\title{
Light emission and excitonic effect of boron nitride nanotubes observed by photoluminescent spectra
}

\author{
Hua Chen ${ }^{a}$, Ying Chen ${ }^{a}$, Yun Liu ${ }^{b, *}$, Chao-Nan $\mathrm{Xu}^{\mathrm{c}}$, Jim S. Williams ${ }^{\mathrm{a}}$ \\ ${ }^{a}$ Research School of Physical Science and Engineering, Australian National University, ACT 0200, Australia \\ ${ }^{\mathrm{b}}$ Research School of Chemistry, Australian National University, ACT 0200, Australia \\ ${ }^{\mathrm{c}}$ National Institute of Advanced Industrial Science and Technology (AIST), Kyushu, Shuku 807-1, Tosu, Saga 841-0052, Japan
}

Received 17 January 2006; received in revised form 21 February 2006; accepted 15 May 2006

Available online 24 July 2006

\begin{abstract}
Photoluminescent (PL) and optical absorption spectra of high-yield multi-wall BN nanotubes (BNNTs) were systematically investigated at room temperature in comparison with commercial hexagonal BN (h-BN) powder. The direct band gap of the BNNTs was determined to be $5.75 \mathrm{eV}$, just slightly narrower than that of h-BN powder (5.82 eV). Two Frenkel excitons with the binding energy of 1.27 and $1.35 \mathrm{eV}$ were also determined. However, they were not a distinctive characteristic of the BNNTs as reported previously. Observed broad UV-visible-NIR light emission demonstrates the potential of the BNNTs as a nano light source.
\end{abstract}

(C) 2006 Elsevier B.V. All rights reserved.

PACS: 78.55.-m; 78.40.Ri; 71.35.-y

Keywords: Boron nitride; Nanotube; Optical absorption; Photoluminescence; Exciton

\section{Introduction}

Boron nitride nanotubes (BNNTs) have been drawn specific attention as a complementary of the carbon nanotubes (CNTs) for application in nanoelectronic and optoelectronic devices. Hexagonal $\mathrm{BN}(\mathrm{h}-\mathrm{BN})$ has a layered structure (like sheets) with a layered interval of $3.33 \AA$ [1]. When these h-BN sheets are rolled up into the BNNTs, the electronic structure of the BN may undergo a change due to the high curvature [2]. Although the BNNTs were successfully synthesized in 1995 [3] and various synthesis routes were also subsequently reported [4-7], the difficulty of fabricating large quantities of high-yield samples considerably restricted the experimental research towards uncovering genuine electronic structure and associated properties of the BNNTs (such as the direct band gap and excitonic

\footnotetext{
* Corresponding author. Tel.: +61 2 61255408; fax: +61 261250750 .

E-mail address: yliu@rsc.anu.edu.au (Y. Liu).
}

effect). Recent theoretical calculation suggested that the band gap of multilayer BNNTs should be reduced about $0.6 \mathrm{eV}[8]$ with respect to h-BN $(5.8 \mathrm{eV})[9,10]$, due to the appearance of $\mathrm{sp}^{3}$ hybridization caused by the large degree of curvature. However, Rubio [11,12] claimed that the band gap of the BNNTs was approximately equal to that of h-BN, nearly independent of their radius and chirality, except for a band gap narrowing in thin NTs (diameter $<\sim 0.8 \mathrm{~nm}$ ) as a result of $\operatorname{sp}^{3}(\pi)$ hybridization induced by the high curvature. Other theoretical calculations [1315], e.g. the tight-binding method or ab initio calculation, indicated that the band gap of the BNNTs was between 5.5 and $6 \mathrm{eV}$. An obviously wide diversity in theoretical prediction of the band gap of the BNNTs necessitates direct experimental clarification.

In addition, more recent calculations predicted that the excitonic effect was stronger in the BNNTs than in the CNTs [16,17]. Lauret reported [18] optical transition in the BNNTs via analysizing optical absorption. The author concluded the existence of distinctive exciton in BNNTs 
with an absorption centred at $4.45 \mathrm{eV}$. The author also predicted the splitting of this exciton in terms of a "selftrapping effect". It is also essential to characterize related features of high quality BNNTs in order to further confirm the existence of Frenkel excitons and to determine the band gap of the BNNTs through a carefully integrated investigation of photoluminescence and optical absorption of the high-yield BNNTs. We believe that this work will benefit the application development of the BNNTs, and simultaneously clarify (and/or provide some evidences for) some theoretical predictions from an experimental point of view.

\section{Experimental}

The BNNTs were synthesized using a ball millingannealing process [4-7] with iron catalysts. This process can fabricate a large quantity of high-yield BNNTs. Fig. 1 shows a typical image of as-synthesised BNNTs taken by Field Emission Scanning Electron Microscopy (FESEM). As observed, the produced nanotubes are homogenous with a diameter of approximate $100 \mathrm{~nm}$. These high-yield bamboo-like nanotubes assure that the collected PL spectral data can reflect genuine information about BNNTs, and subsequently revealing the real electronic structure and optical properties of the BNNTs.

In this work, the commercial h-BN powder (Aldrich, $99 \%, 1 \mu \mathrm{m}$ ) was also studied as a reference.

The optical absorption spectra of the samples were collected using a UV-Vis-NIR spectrophotometer (CARY-5) at room temperature. Their photoluminescent properties were carefully measured by a spectrofluorometer (FP6500 ; Jasco Inc.). The resolution is $1 \mathrm{~nm}$ for both excitation and emission spectra.

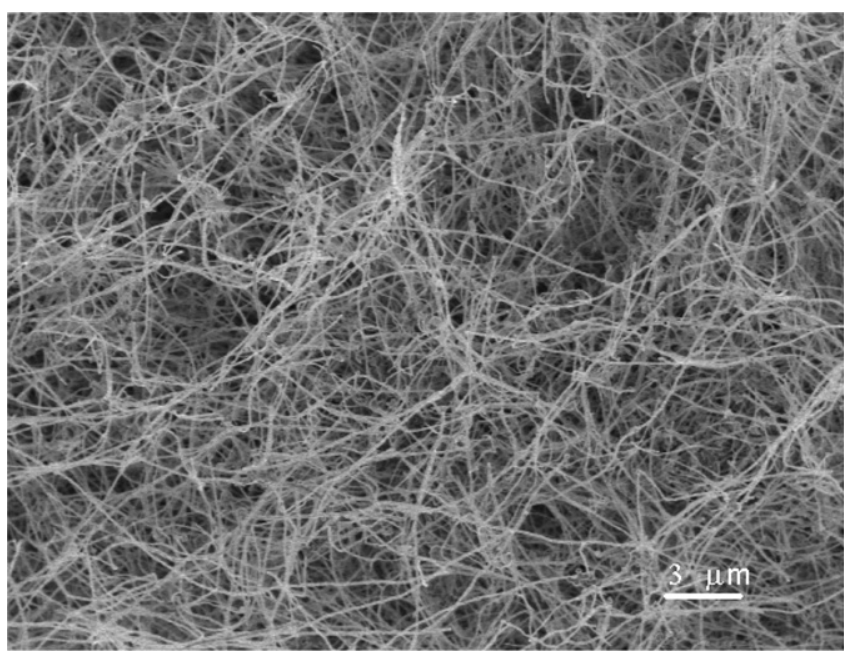

Fig. 1. FESEM image of as-synthesised BNNTs using a ball-milling and annealing process.

\section{Results and discussion}

\subsection{Optical absorption}

Fig. 2 shows the optical absorption spectra of both h-BN powder and BNNTs. The observed absorption edge definitely indicates that h-BN and BNNTs have an identical band gap larger than $5.5 \mathrm{eV}$, greatly consistent with Rubio's prediction [11]. The accurate band gap cannot be determined by these spectra because of the instrumental wavelength limit. A peak centred at about $4.0 \mathrm{eV}$, adjacent to its absorption edge, is found in the h-BN. In contrast, a very broad absorption is found in the BNNTs that slowly increases from $2.0 \mathrm{eV}$ to its absorption edge, instead of an absorption peak. The difference between the h-BN and BNNTs in the spectra suggests the existence of a high density of exciton energy levels and/or many deep impurity energy levels. It is difficult to determine what kind of impure energy levels appear in the sample at this stage. However, the appearance of the excitons is certain in the boron nitride based on various simulation models and observations, e.g. optical absorption spectra from the hBN powder and BNNTs [16-18]. The relative intensity of the h-BN absorption peak is obviously weaker than the absorption intensity of the BNNTs, demonstrating more energy levels below the bottom of conductive band in the BNNTs. The formation of these energy levels probably arises from highly structural distortion caused by the high curvature in BNNTs.

\subsection{Near UV emission and intrinsic excitation}

Fig. 3 shows typical photoluminescent spectra collected from both h-BN powder and BNNTs at room temperature. A strong peak centred at $5.82 \mathrm{eV}(213 \mathrm{~nm})$ was found in the excitation spectrum of the h-BN powder. This value is extremely consistent with previously reported direct band gap of the h-BN [10]. Hence, this excitation peak is

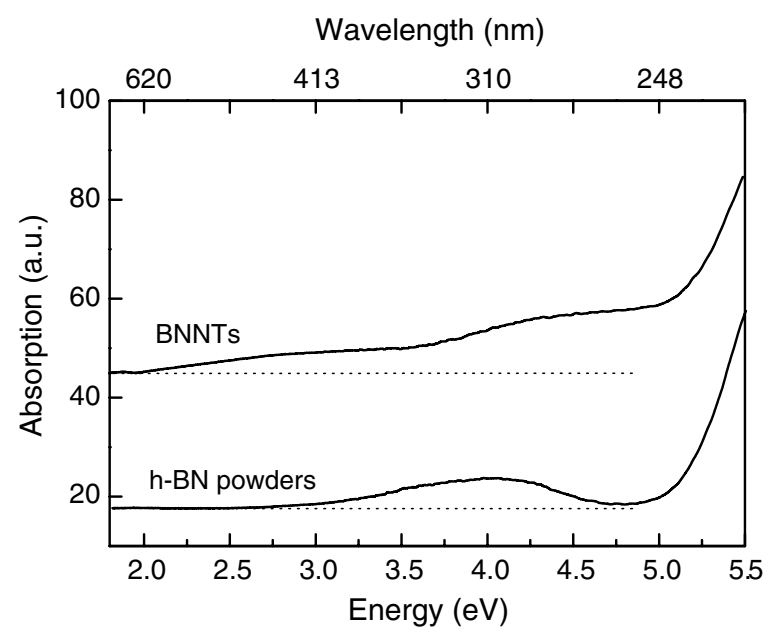

Fig. 2. Optical absorption spectra of the h-BN powder and the BNNTs at room temperature. 


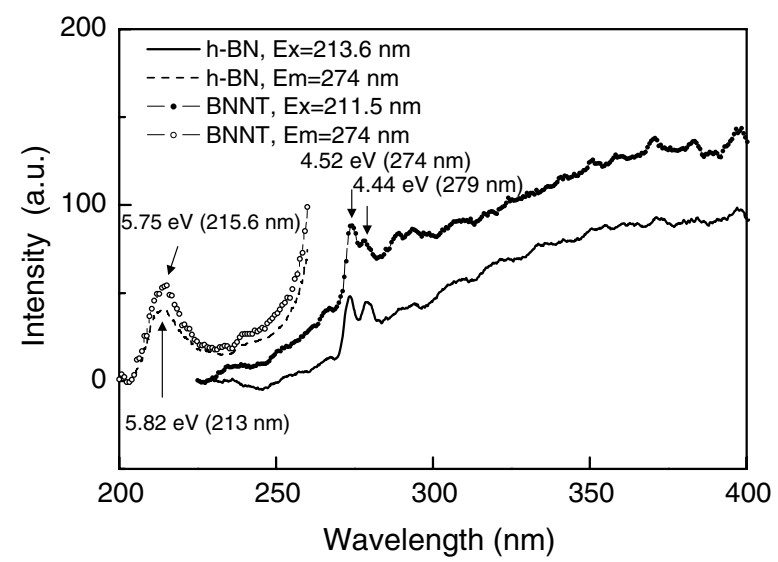

Fig. 3. Near-UV emission and intrinsic excitation spectra of the h-BN powder and the BNNTs.

ascribed to the intrinsic excitation of $\mathrm{h}-\mathrm{BN}$ powder. The $5.82 \mathrm{eV}$ peak is determined to be the direct band gap of the commercial h-BN powders used in this work. Correspondingly, the excitation peak centred at $5.75 \mathrm{eV}$ $(215.6 \mathrm{~nm})$ is the direct band gap of the BNNTs. Obviously, both direct band gaps are larger than $5.5 \mathrm{eV}$, coinciding with the observed optical absorption as shown in Fig. 2. There is only an $0.07 \mathrm{eV}$ reduction from powder to nanotubes, in good agreement with Rudio's prediction $[11,12]$, but far away from some predictions of other authors (they claimed that the difference of band gaps between the h-BN powders and BNNTs should be $0.6 \mathrm{eV}$ [8] or more [9]). In terms of their structural features, we found they were not contradictory. The h-BN powder has a layered structure and the distance between the layers is $3.33 \AA$. When these sheets are rolled up to form a nanotube (multi-walls), the distance between the walls still remains $3.3 \AA$ [19]. Therefore, there is no reason to considerably change the band gap of the BNNTs as observed in this work. In addition, the diversity of the band gap of the BNNTs in calculations is found from different assumptions. In most cases, the calculations are carried out based on a single wall BNNT with a tube diameter of less than one nanometer $[17,20]$, where the nano size effect and $\mathrm{sp}^{3}$ hybridizing effect may predominate the BNNT and as a result, leading to the significant decrease of its band gap. In our case, the diameter of multilayer BNNTs is much larger. Therefore, the observed band gap of the BNNTs is approximately equal to that of the h-BN powder.

Nevertheless, the high curvature must cause some strains in this "roll-up" structure from a crystallographic point of view. These strains would create a large amount of additional energy levels within the band gap, giving rise to a broad absorption in the BNNTs as shown in Fig. 2. In addition, the asymmetry of the intrinsic excitation peak of BNNTs (see Fig. 3) also reflects some distortions as well.

Fig. 3 also presents the emission spectra of both powder and nanotubes under an intrinsic excitation. As a result, a strong broad near-UV light emission is observed in both samples, in good agreement with observed optical absorption as shown in Fig. 2. For the BNNTs, intrinsic excitation directly contributes to a strong light emission owing to the strong absorption observed in Fig. 2. Two distinct peaks were found from the emission spectra. They are localized at $274 \mathrm{~nm}(4.52 \mathrm{eV})$ and at $279 \mathrm{~nm}(4.44 \mathrm{eV})$, respectively. It is of worthy note that the corresponding energy levels are actually very close to the excitonic line (around $4.45 \mathrm{eV}$ ) described by Lauret et al. [18]. Lauret further predicted that "this line may decompose into two lines centred at 4.4 and $4.5 \mathrm{eV}$ " in terms of his observation on optical absorption. Correspondingly, we can determine that the two observed peaks in this work are related to the excitonic effect. The binding energies of these two excitons can be determined according to the $E_{\text {exciton }}=$ $E_{\mathrm{g}}-E_{\text {emission. }}$. These are 1.30 and $1.38 \mathrm{eV}$ for h-BN powder and, 1.27 and $1.35 \mathrm{eV}$ for the BNNTs, respectively. Furthermore, the exciton binding energy is larger than $1 \mathrm{eV}$, hence these excitons belong to Frenkel excitons.

The formation of the exciton arises from the binding of a hole and an electron (excited by intrinsic excitation photons). The excitons localise their binding energies just below the bottom of the conductive band. In general, the covalent binding within a molecule is stronger in comparison with the Van Der Waals binding between molecules [21], so that the excitons created by the B-N Coulomb interaction are Frenkel excitons. Also, note that as the negative ions have lower electronic excitation levels than the positive ions [21], the difference between boron and nitrogen atoms creates two excitons with different binding energies, corresponding to the $\mathrm{N}-\mathrm{B}$ and $\mathrm{B}-\mathrm{N}$. We do observe two excitons in both h-BN and BNNTs with slightly different emission intensities. Therefore, the existence of two excitons is not a distinctive feature of the BNNTs. Moreover, the BNNT are a one-dimensional material. Stronger anisotropy of the BNNTs may vary the relative emission intensities of the excitons as shown in Fig. 3.

\subsection{White light emission}

The difference of optical absorption spectra (see Fig. 2) between the h-BN and BNNTs illustrates that there are more energy levels in the BNNTs. Compared to the high curvature of BNNTs, the h-BN has less distortion. Therefore, we believe that BNNTs have more defect energy levels than the h-BN, which can create a large range of optical absorption, and thus lead to white light emission as shown in Fig. 3. Note that the light emission still increases at $400 \mathrm{~nm}$ in Fig. 3, we are curious about what would happen in the visible light range. The excitation light wavelength was chosen to be $245 \mathrm{~nm}(5.06 \mathrm{eV})$, determined by the absorption edge in Fig. 2. A broad white light emission is observed in both the h-BN and BNNTs samples in the visible light range (Fig. 4). The BNNTs show much stronger intensity. From Fig. 4, the emission of the h-BN powder peaked at around $400 \mathrm{~nm}$. We cannot determine the emission peak of BNNTs due to the harmonic effect limited by the instrument used, but the collected PL emission defi- 


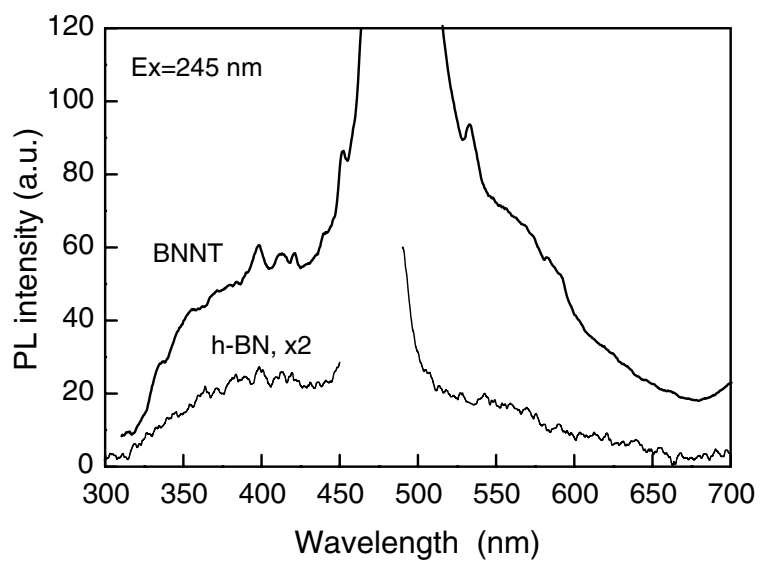

Fig. 4. Photoluminescent spectra of the h-BN powder and the BNNTs under an excitation wavelength of $245 \mathrm{~nm}$.

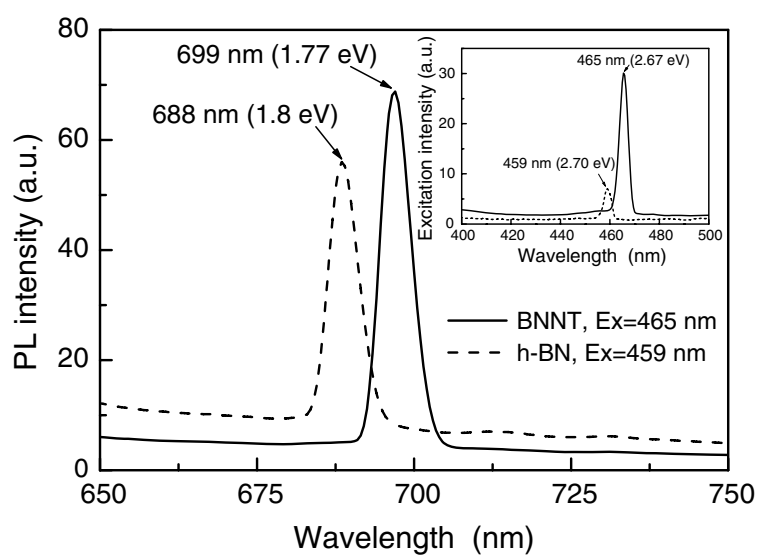

Fig. 5. Photoluminescent spectra of the h-BN powder and the BNNTs under a visible light excitation. An insert is the associated excitation spectra.

nitely indicates a red-shift (around $100 \mathrm{~nm}$ ) of the BNNTs in contrast to the h-BN powder. In the BNNTs, a few PL peaks can also be picked at: $398 \mathrm{~nm}, 412 \mathrm{~nm}, 420 \mathrm{~nm}$, $450 \mathrm{~nm}$ and $533 \mathrm{~nm}$, but it is difficult to determine where they come from.

\subsection{Near infrared light emission}

Fig. 5 shows both excitation and emission spectra of the $\mathrm{h}-\mathrm{BN}$ and BNNTs under a visible light excitation. One excitation peak is distinctly found in both samples, corresponding to $459 \mathrm{~nm}(2.70 \mathrm{eV})$ for the h-BN powder and $465 \mathrm{~nm}(2.67 \mathrm{eV})$ for the BNNTs, respectively. The near infrared emission is excited under these excitation lights. The emission peaks are centred at $1.8 \mathrm{eV}(688 \mathrm{~nm})$ for the h-BN powder and $1.77 \mathrm{eV}(699 \mathrm{~nm})$ for the BNNTs. Again, the red-shift phenomena due to the formation of nanotubes are observed. In the light of previous theoretical calculation, it may also be ascribed to excitonic effect as well, but we need more evidence to prove it. The associated work is in progress.

\section{Conclusion}

This work systematically investigates the PL and optical absorption spectra of high-yield multi-wall BNNTs. The results show that (1) the direct band gap of multi-wall BNNTs with a diameter of approximately $100 \mathrm{~nm}$ is $5.75 \mathrm{eV}$, just slightly narrower than the h-BN powder $(5.82 \mathrm{eV}) ;(2)$ the BNNTs present stronger and broader UV-visible-NIR light emission in comparison with h-BN powder; and (3) two split excitons are found in the BNNTs with exciton binding energy of 1.27 and $1.35 \mathrm{eV}$, respectively, which are not a distinctive feature of the BNNTs. The observed light emission implies some potential for the BNNTs to be used as nano light sources.

\section{Acknowledgements}

Authors (Y. Liu and Y. Chen) acknowledge financial support from the Australian Research Council (ARC) in the form of the ARC Discovery Grant.

\section{References}

[1] R. Pease, Acta Cryst. 5 (1952) 356.

[2] J. Wu, W.-Q. Han, W. Walukiewicz, J.W. Ager, W. Shan, E.E. Haller, A. Zettl, Nano Lett. 4 (2004) 647.

[3] N.G. Chopra, R.J. Luyken, K. Cherrey, V.H. Crespi, M.L. Cohen, S.G. Louie, A. Zettl, Science 269 (1995) 966.

[4] K. Suenaga, C. Colliex, N. Demoncy, A. Loiseau, H. Pascard, F. Willaime, Science 278 (1997) 653.

[5] Y. Zhang, H. Gu, K. Suenaga, S. Iijima, Chem. Phys. Lett. 279 (1997) 264.

[6] R. Ma, Y. Bando, T. Sato, Adv. Mater. 14 (2002) 366.

[7] Y. Chen, J. Fitz Gerald, J.S. Williams, S. Bulcock, Chem. Phys. Lett. 299 (1999) 260.

[8] G.G. Fuentes, E. Borowiak-Palen, T. Pichler, X. Liu, A. Graff, G. Behr, R.J. Kalenczuk, M. Knupfer, J. Fink, Phys. Rev. B 67 (2003) 035429.

[9] S. Okada, S. Saito, A. Oshiyama, Phys. Rev. B 65 (2002) 165410.

[10] A. Zunger, A. Katzir, A. Halperin, Phys. Rev. B 13 (1976) 5560.

[11] A. Rubio, J.L. Corkill, M.L. Cohen, Phys. Rev. B 49 (1994) 5081.

[12] X. Blase, A. Rubio, S. Louie, M. Cohen, Europhys. Lett. 28 (1994) 335 .

[13] A.G. Marinopoulos, L. Wirtz, A. Marini, V. Olevano, A. Rubio, L. Reining, Appl. Phys. A 78 (2004) 1157.

[14] M.-F. Ng, R.Q. Zhang, Phys. Rev. B 69 (2004) 115417.

[15] O. Stephan, Proceedings of the Institute of Physics Electron Microscopy and Analysis Group, vol. 179, University of Oxford, Institute of Physics Publishing, Bristol, 2003, p. 437.

[16] L. Wirtz, A. Marini, A. Rubio, cond-mat 1, 2005, 0508701.

[17] C.D. Spataru, S. Ismail-Beigi, L.X. Benedict, S.G. Louie, Phys. Rev. Lett. 92 (2004) 077402.

[18] J.S. Lauret, R. Arenal, F. Ducastelle, A. Loiseau, M. Cau, B. AttalTretout, E. Rosencher, L. Goux-Capes, Phys. Rev. Lett. 94 (2005) 037405 .

[19] H.C. Choi, S.Y. Bae, W.S. Jang, J. Park, H.J. Song, H.-J. Shin, J. Phys. Chem. B 109 (2005) 7007.

[20] F. Wang, G. Dukovic, L.E. Brus, T.F. Heinz, Science 308 (2005) 838

[21] C. Kittel, Introduction to Solid State Physics, John Wiley and Sons Inc., New York, 1996. 